\title{
Transverse momentum distributions and nuclear effects
}

\author{
Emanuele Pace ${ }^{1, a}$, Alessio Del Dotto ${ }^{2}$, Leonid Kaptari ${ }^{3,4}$, Matteo Rinaldi ${ }^{4}$, Giovanni Salmè ${ }^{5}$, and Sergio Scopetta ${ }^{4}$ \\ ${ }^{1}$ Dipartimento di Fisica, Università di Roma “Tor Vergata” and INFN, Roma 2, Italy \\ ${ }^{2}$ Dipartimento di Fisica, Università di Roma Tre and INFN, Roma 3, Italy \\ ${ }^{3}$ Bogoliubov Lab. Theor. Phys., 141980, JINR, Dubna, Russia \\ ${ }^{4}$ Dipartimento di Fisica e Geologia, Università di Perugia and INFN, Sezione di Perugia, Italy \\ ${ }^{5}$ INFN Sezione di Roma, Italy
}

\begin{abstract}
A distorted spin-dependent spectral function for ${ }^{3} \mathrm{He}$ is considered to take care of the final state interaction in the extraction of the quark transverse-momentum distributions in the neutron from semi-inclusive deep inelastic electron scattering off polarized ${ }^{3} \mathrm{He}$ at finite momentum transfers. The generalization of the analysis in a Poincaré covariant framework within the light-front dynamics is outlined. The definition of the light-front spin-dependent spectral function for a $\mathrm{J}=1 / 2$ system, as the nucleon, allows us to show that within the light-front dynamics and in the valence approximation only three of the six leading twist T-even transversemomentum distributions are independent.
\end{abstract}

\section{Introduction}

Information on the quark orbital angular momentum, $L_{q}$, of the proton can be obtained from the quark transverse momentum distributions (TMDs) [1], that can be accessed through non forward processes, as semi-inclusive deep inelastic electron scattering (SiDIS). In particular the single spin asymmetries (SSAs) allow one to experimentally distinguish the Sivers and the Collins asymmetries [2, 3], expressed in terms of different TMDs and fragmentation functions (ff) [1,4]. A large Sivers asymmetry was measured in $\vec{p}\left(e, e^{\prime} \pi\right) x$ [5] and a small one in $\vec{D}\left(e, e^{\prime} \pi\right) x$ [6], showing a strong flavor dependence of TMDs. This puzzle has attracted a great interest in obtaining new information on the neutron TMDs. To clarify the flavour dependence of the quark transverse momentum distributions, high precision experiments involving both protons and neutrons are needed [7].

In Ref. [8] the possibility to extract information on the neutron TMDs from experimental measurements of the SSAs on ${ }^{3} \mathrm{He}$ was proposed. To obtain a reliable information one has to take carefully into account : i) the nuclear structure of ${ }^{3} \mathrm{He}$, ii) the interaction in the final state (FSI) between the observed pion and the remnant debris, and iii) the relativistic effects.

The present paper reports on our efforts about the above items. In Sect. II the possibility to extract the neutron SSAs from ${ }^{3} \mathrm{He}$ SSAs in plane wave impulse approximation (PWIA) [4] is briefly summarized; in Sect. III preliminary results about the effects of FSI are illustrated $[9,10]$; in Section IV the possibility to study the relativistic effects through a light-front (LF) spin-dependent spec-

\footnotetext{
ae-mail: pace@roma2.infn.it
}

tral function for ${ }^{3} \mathrm{He}$ is outlined [11-13], and eventually in Sect. V the relations between the LF spin-dependent spectral function for the quarks in a nucleon, the quark-quark correlator and the TMDs are studied [11-14]. In Sect. VI conclusions are drawn.

\section{Polarized ${ }^{3} \mathrm{He}$ target as a polarized neutron target}

A polarized ${ }^{3} \mathrm{He}$ is an ideal target to study the neutron, since at a $90 \%$ level a polarized ${ }^{3} \mathrm{He}$ is equivalent to a polarized neutron. Dynamical nuclear effects in inclusive deep inelastic electron scattering ${ }^{3} \vec{H} e\left(e, e^{\prime}\right) X$ (DIS) were evaluated [15] with a realistic ${ }^{3} \vec{H} e$ spin-dependent spectral function, $P_{\sigma, \sigma}^{\tau}\left(\mathbf{p}, E, S_{H e}\right)$, with $\mathbf{p}$ the initial nucleon momentum in the laboratory frame and $E$ the missing energy [16]. It was found that the formula

$$
A_{n} \simeq \frac{1}{p_{n} f_{n}}\left(A_{3}^{\exp }-2 p_{p} f_{p} A_{p}^{\exp }\right)
$$

can be safely adopted to extract the neutron information from ${ }^{3} \mathrm{He}$ and proton data. This formula is actually widely used by experimental collaborations (see, e.g. Ref. [17]). The nuclear effects are hidden in the proton and neutron "effective polarizations", $p_{p(n)}$. With the AV18 nucleonnucleon interaction [18] the values $p_{p}=-0.023, p_{n}=$ 0.878 were obtained [4]. The quantities $f_{p(n)}$ in Eq. (1) are the dilution factors.

To investigate if an analogous formula can be used to extract the SSAs, in [4] the processes ${ }^{3} \vec{H} e\left(e, e^{\prime} \pi^{ \pm}\right) X$ were evaluated in the Bjorken limit and in PWIA, i.e. the final 

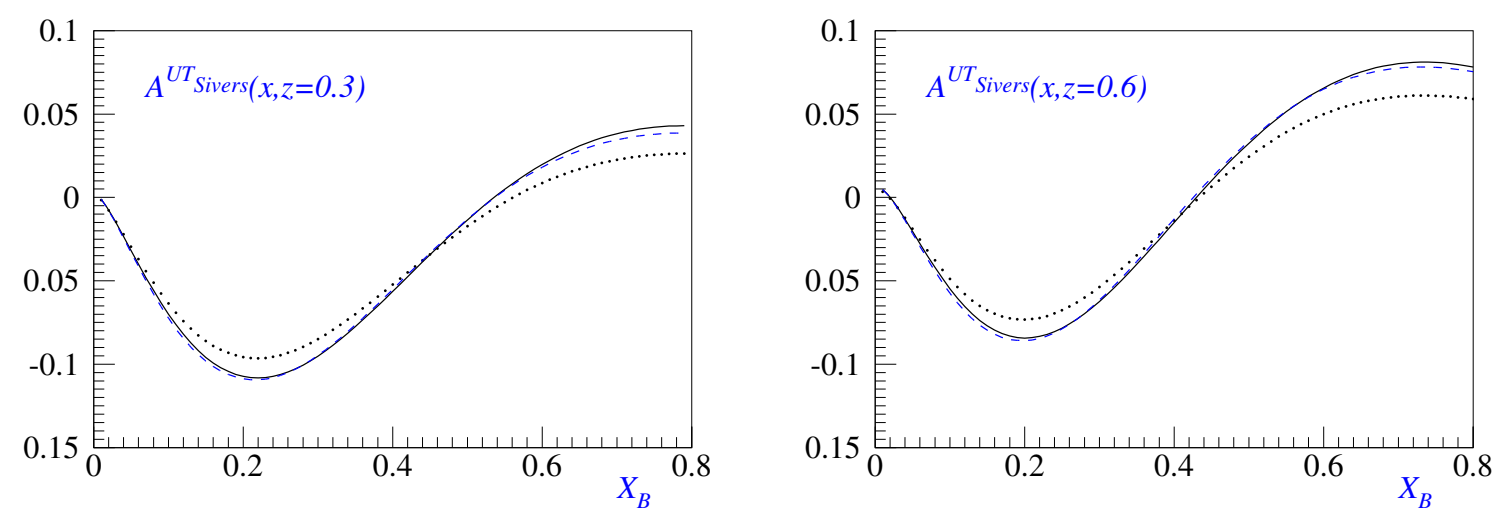

Figure 1. Sivers asymmetry. Full line: neutron asymmetry of the adopted model; dotted line: neutron asymmetry extracted from the calculated ${ }^{3} \mathrm{He}$ asymmetry neglecting the proton polarization contribution: $\bar{A}_{n} \simeq \frac{1}{f_{n}} A_{3}^{\text {calc }}$; dashed line: neutron asymmetry extracted from the calculated ${ }^{3} \mathrm{He}$ asymmetry taking into account the nuclear structure through Eq. (1) (after [4]).

state interaction (FSI) was considered only within the twonucleon spectator pair which recoils. In such a framework, SSAs for ${ }^{3} \mathrm{He}$ involve convolutions of $P_{\sigma, \sigma^{\prime}}^{\tau}\left(\mathbf{p}, E, S_{H e}\right)$, with TMDs and fragmentation functions. Ingredients of the calculations were: i) a realistic $P_{\sigma, \sigma^{\prime}}^{\tau}\left(\mathbf{p}, E, S_{H e}\right)$ for ${ }^{3} \mathrm{He}$ [16], obtained using the AV18 interaction; ii) parametrizations of data for TMDs and ff, whenever available; iii) models for the unknown TMDs and ff. As shown in Fig. 1, in the Bjorken limit the extraction procedure through the formula successful in DIS works nicely for the Sivers SSA and the same was shown to occur for the Collins SSA [4]. The generalization of Eq. (1) to extract Sivers and Collins asymmetries from ${ }^{3} \mathrm{He}$ and proton asymmetries was recently used by experimental collaborations [19, 20].

\section{Distorted spin-dependent spectral function for ${ }^{3} \mathrm{He}$}

In SiDIS experiments off ${ }^{3} \mathrm{He}$, the relative energy between the spectator $(A-1)$ system and the system composed by the detected pion and the remnant debris (see Fig. 2) is a few $\mathrm{GeV}$ and FSI can be treated through a generalized eikonal approximation (GEA) [21]. The GEA was already succesfully applied to unpolarized SiDIS in Ref. [22]. The FSI effects to be considered are due to the propagation of the debris, formed after the $\gamma^{*}$ absorption by a target quark, and the subsequent hadronization, both of them influenced by the presence of a fully interacting $(A-1)$ spectator system (see Fig. 2).

The key quantity to introduce the FSI effects is the distorted spin-dependent spectral function, whose relevant part in the evaluation of SSAs is :

$$
\mathcal{P}_{\|}^{P W I A(F S I)}=O_{\frac{1}{2} \frac{1}{2}}^{I A(F S I)}-O_{-\frac{1}{2}-\frac{1}{2}}^{I A(F S I)}
$$

with:

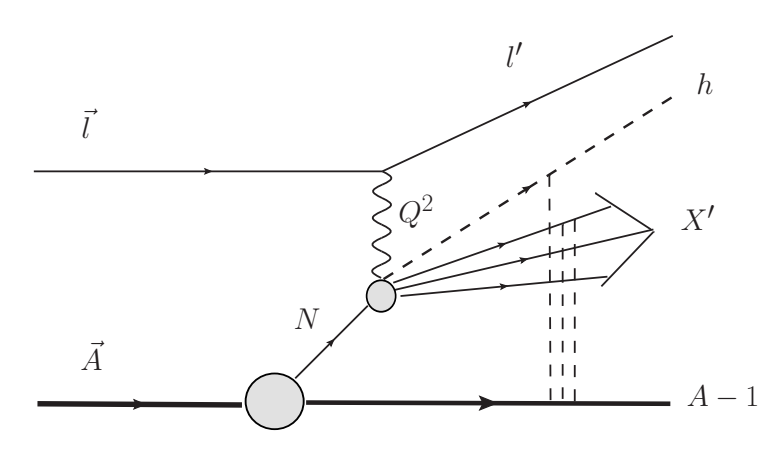

Figure 2. Interaction between the $(A-1)$ spectator system and the debris produced by the absorption of a virtual photon by a nucleon in the nucleus.

$$
\begin{aligned}
& O_{\lambda \lambda^{\prime}}^{I A}(\vec{p}, E)= \\
& =£ d \epsilon_{A-1}^{*} \rho\left(\epsilon_{A-1}^{*}\right)\left\langle S_{A}, \mathbf{P}_{A} \mid \Phi_{\epsilon_{A-1}^{*}}, \lambda^{\prime}, \vec{p}\right\rangle \times \\
& \left\langle\Phi_{\epsilon_{A-1}^{*}}, \lambda, \vec{p} \mid S_{A}, \mathbf{P}_{A}\right\rangle \delta\left(E-B_{A}-\epsilon_{A-1}^{*}\right)
\end{aligned}
$$

and

$$
\begin{aligned}
& O_{\lambda \lambda^{\prime}}^{F S I}(\vec{p}, E)= \\
& =\oint d \epsilon_{A-1}^{*} \rho\left(\epsilon_{A-1}^{*}\right)\left\langle S_{A}, \mathbf{P}_{A} \mid\left(\hat{S}_{G l}\right)\left\{\Phi_{\epsilon_{A-1}^{*}}, \lambda^{\prime}, \vec{p}\right\}\right\rangle \times \\
& \left\langle\left(\hat{S}_{G l}\right)\left\{\Phi_{\epsilon_{A-1}^{*}}^{*}, \lambda, \vec{p}\right\} \mid S_{A}, \mathbf{P}_{A}\right\rangle \delta\left(E-B_{A}-\epsilon_{A-1}^{*}\right)
\end{aligned}
$$

where $\rho\left(\epsilon_{A-1}^{*}\right)$ is the proper density of the $(A-1)$-system states with intrinsic energy $\epsilon_{A-1}^{*}$, and $\left|S_{A}, \mathbf{P}_{A}\right\rangle$ is the ground 
state of the $A$-nucleons nucleus with polarization $S_{A}$. The quantity $\hat{S}_{G l}$ is the Glauber operator:

$$
\begin{array}{r}
\hat{S}_{G l}\left(\mathbf{r}_{1}, \mathbf{r}_{2}, \mathbf{r}_{3}\right)= \\
\prod_{i=2,3}\left[1-\theta\left(z_{i}-z_{1}\right) \Gamma\left(\mathbf{b}_{1}-\mathbf{b}_{i}, z_{1}-z_{i}\right)\right]
\end{array}
$$

and $\Gamma\left(\mathbf{b}_{1 i}, z_{1 i}\right)$ the generalized profile function:

$$
\Gamma\left(\mathbf{b}_{1 i}, z_{1 i}\right)=\frac{(1-i \alpha) \sigma_{e f f}\left(z_{1 i}\right)}{4 \pi b_{0}^{2}} \exp \left[-\frac{\mathbf{b}_{1 i}^{2}}{2 b_{0}^{2}}\right],
$$

where $\mathbf{r}_{1 i}=\left\{\mathbf{b}_{1 i}, \mathbf{z}_{1 i}\right\}$ with $\mathbf{z}_{1 i}=\mathbf{z}_{1}-\mathbf{z}_{i}$ and $\mathbf{b}_{1 i}=\mathbf{b}_{1}-\mathbf{b}_{i}$.

The models for the profile function, $\Gamma\left(\mathbf{b}_{1 i}, z_{1 i}\right)$, and for the effective cross section, $\sigma_{e f f}\left(z_{1 i}\right)$, as well as the values of the parameters $\alpha$ and $b_{0}$ are the ones exploited in Ref. [22] to nicely describe the data measured at JLab [23], corresponding to the unpolarized spectator SiDIS off the deuteron. In particular, $\sigma_{\text {eff }}\left(z_{1 i}\right)$ is the cross section for the interaction of the formed hadrons (assumed to be a nucleon and a few pions) with the remnant nucleons, and is given by the sum of two contributions: i) the nucleon-nucleon cross section and ii) and the product of the nucleon-pion cross section times the effective number of pions which are produced (cf Ref. [24] for the explicit evaluation). The parameter $\alpha$ in Eq. (6) is the ratio of the real to the imaginary part of the forward scattering amplitude of the process. In our calculation it has been taken constant, namely, $\alpha=0.5$, following [22]. Finally, the slope parameter, $b_{0}$, is determined by the optical theorem, which yields $b_{0}^{2}=\sigma_{e f f}^{2}\left(1+\alpha^{2}\right) /\left[16 \pi \sigma^{e l}\right]$, where $\sigma^{e l}$ is the elastic part of the total effective cross section $\sigma_{e f f}$.

It occurs that $\mathcal{P}_{\|}^{P W I A}$ and $\mathcal{P}_{\|}^{F S I}$ can be very different, but the relevant observables for the SSAs involve integrals, dominated by the low momentum region, where the FSI effects on $\mathcal{P}_{\|}$are minimized and the spectral function is large [22]. As a consequence the effective nucleon polarizations change from $p_{p}=-0.023, p_{n}=0.878$ to $p_{p}^{F S I}=-0.026, p_{n}^{F S I}=0.76$, where

$$
p_{p(n)}^{F S I}=\int d \epsilon_{S} \int d \mathbf{p} \operatorname{Tr}\left[\mathbf{S}_{H e} * \sigma P_{F S I}^{p(n)}\left(\vec{p}, E, S_{H e}\right)\right],
$$

with $P_{F S I}^{p(n)}\left(\vec{p}, E, S_{H e}\right)$ the distorted spin-dependent spectral function, defined in terms of the overlaps of Eq. (4) [10]. Then $p_{p(n)}$ with and without the FSI differ by $10-15 \%$. Actually, one has also to consider the effect of the FSI on dilution factors. We have found, in a wide range of kinematics typical for the experiments at JLAB $[8,25]$, that the product of polarizations and dilution factors changes very little [10]. Indeed the effects of FSI in the dilution factors and in the effective polarizations are found to compensate each other to a large extent and the usual extraction appears to be safe :

$$
\begin{gathered}
A_{n} \simeq \frac{1}{p_{n}^{F S I} f_{n}^{F S I}}\left(A_{3}^{e x p}-2 p_{p}^{F S I} f_{p}^{F S I} A_{p}^{e x p}\right) \simeq \\
=\frac{1}{p_{n} f_{n}}\left(A_{3}^{e x p}-2 p_{p} f_{p} A_{p}^{\text {exp }}\right)
\end{gathered}
$$

\section{Light-front description of deep inelastic scattering off ${ }^{3} \mathrm{He}$}

In [4] the calculation was performed within a non relativistic approach for the spectral function, but with the correct relativistic kinematics in the Bjorken limit. For an accurate description of SiDIS processes, the role played by relativity has to be fully investigated: it will become even more important with the upgrade of JLab @ $12 \mathrm{GeV}$.

To study relativistic effects in the actual experimental kinematics, we adopted [11] the Relativistic Hamiltonian Dynamics (RHD) introduced by Dirac [26]. Indeed the RHD of an interacting system with a fixed number of on-massshell constituents, with the Bakamijan-Thomas construction of the Poincaré generators [27], is fully Poincaré covariant. We choose the LF form of RHD. It has a subgroup structure of the LF boosts and allows a separation of the intrinsic motion from the global one, which is very important for the description of DIS, SiDIS and deeply virtual Compton scattering (DVCS) processes, and allows a meaningful Fock expansion.

The key quantity to consider in SIDIS processes is the LF relativistic spectral function, $\mathcal{P}_{\sigma^{\prime} \sigma}^{\tau}\left(\tilde{\kappa}, \epsilon_{S}, S_{H e}\right)$, with $\tilde{\kappa}$ an intrinsic nucleon momentum and $\epsilon_{S}$ the energy of the twonucleon spectator system. The LF spectral function is very useful also for other studies (e.g., for nuclear generalized parton distributions (GPDs), where final states have to be properly boosted).

In IA the LF hadronic tensor for the ${ }^{3} \mathrm{He}$ nucleus is:

$$
\begin{array}{r}
\mathcal{W}^{\mu v}\left(Q^{2}, x_{B}, z, \tau_{h}, \hat{\mathbf{h}}, S_{H e}\right) \propto \sum_{\sigma \sigma^{\prime}, \tau} \sum_{\epsilon_{S}^{\min }}^{\epsilon_{S}^{\max }} d \epsilon_{S} \\
\times \int_{M_{N}^{2}}^{\left(M_{X}-M_{S}\right)^{2}} d M_{f}^{2} \int_{\xi l}^{\xi_{u}} \frac{d \xi}{\xi^{2}(1-\xi)(2 \pi)^{3}} \int_{p_{\perp}^{m}}^{p_{\perp}^{M}} \frac{d \mathbf{p}_{\perp}}{\sin \theta} \\
\times\left(p^{+}+q^{+}-h^{+}\right) w_{\sigma \sigma^{\prime}, \tau}^{\mu v}\left(\tilde{\mathbf{q}}, \tau_{h}, \tilde{\mathbf{h}}, \tilde{\mathbf{p}}\right) \mathcal{P}_{\sigma^{\prime} \sigma}^{\tau}\left(\tilde{\kappa}, \epsilon_{S}, S_{H e}\right)
\end{array}
$$

where $z=P_{H e} \cdot h /\left(P_{H e} \cdot q\right), \tilde{\mathbf{v}}=\left\{v^{+}=v^{0}+v^{3}, \mathbf{v}_{\perp}\right\}, \tilde{\mathbf{q}}$ is the virtual photon momentum, $\tilde{\mathbf{h}}$ the detected pion momentum, $M_{f}$ the mass of the remnant (X', see Fig. 2), $\xi=p^{+} / P_{H e}^{+}, \theta$ is the angle between $\mathbf{p}_{\perp}$ and $\left(\mathbf{q}_{\perp}-\mathbf{h}_{\perp}\right)$, $w_{\sigma \sigma^{\prime}, \tau}^{\mu \nu}\left(\tilde{\mathbf{q}}, \tau_{h}, \tilde{\mathbf{h}}, \tilde{\mathbf{p}}\right)$ the nucleon hadronic tensor.

The LF nuclear spectral function, $\mathcal{P}_{\sigma^{\prime} \sigma}^{\tau}\left(\tilde{\kappa}, \epsilon_{S}, S_{H e}\right)$, is defined in terms of LF overlaps [14] between the ground state of a polarized ${ }^{3} \mathrm{He}$ and the cartesian product of an interacting state of two nucleons with energy $\epsilon_{S}$ and a plane wave for the third nucleon. Within a reliable approximation [14] it can be given in terms of the unitary Melosh Rotations, $D^{\frac{1}{2}}\left[\mathcal{R}_{M}(\tilde{\kappa})\right]$, and the usual instant-form spectral function $P_{\sigma_{1}^{\prime} \sigma_{1}}^{\tau}$ :

$$
\begin{gathered}
\mathcal{P}_{\sigma^{\prime} \sigma}^{\tau}\left(\tilde{\kappa}, \epsilon_{S}, S_{H e}\right) \propto \\
\sum_{\sigma_{1} \sigma_{1}^{\prime}} D^{\frac{1}{2}}\left[\mathcal{R}_{M}^{\dagger}(\tilde{\kappa})\right]_{\sigma^{\prime} \sigma_{1}^{\prime}} P_{\sigma_{1}^{\prime} \sigma_{1}}^{\tau}\left(\mathbf{p}, \epsilon_{S}, S_{H e}\right) D^{\frac{1}{2}}\left[\mathcal{R}_{M}(\tilde{\kappa})\right]_{\sigma_{1} \sigma}
\end{gathered}
$$

Notice that the instant-form spectral funtion $P_{\sigma_{1}^{\prime} \sigma_{1}}^{\tau}\left(\mathbf{p}, \epsilon_{S}, S_{H e}\right)$ is given in terms of three independent functions, $B_{0}, B_{1}, B_{2}$ [16], once parity and t-reversal are imposed:

$$
P_{\sigma_{1}^{\prime} \sigma_{1}}^{\tau}\left(\mathbf{p}, \epsilon_{S}, S_{H e}\right)=\left[B_{0, S_{H e}}^{\tau}(|\mathbf{p}|, E)+\boldsymbol{\sigma} \cdot \mathbf{f}_{S_{H e}}^{\tau}(\mathbf{p}, E)\right]_{\sigma_{1}^{\prime} \sigma_{1}}
$$


Table 1. Proton and neutron effective polarizations within the non relativistic appproach (NR) and preliminary results within the light-front relativistic dynamics approach (LF). First line : longitudinal effective polarizations; second line : transverse effective polarizations.

\begin{tabular}{|l|c|c|c|c|}
\hline & proton NR & proton LF & neutron NR & neutron LF \\
\hline $\int d E d \vec{p} \operatorname{Tr}\left(\mathcal{P}_{\sigma_{z}}\right)_{\vec{S}_{A}=\bar{z}}$ & -0.02263 & -0.02231 & 0.87805 & 0.87248 \\
\hline $\int d E d \vec{p} \operatorname{Tr}\left(\mathcal{P}_{\sigma_{y}}{\overrightarrow{S_{A}}}_{\bar{y}}\right.$ & -0.02263 & -0.02268 & 0.87805 & 0.87494 \\
\hline
\end{tabular}

with

$\mathbf{f}_{S_{H e}}^{\tau}(\mathbf{p}, E)=\mathbf{S}_{H e} B_{1, S_{H e}}^{\tau}(|\mathbf{p}|, E)+\hat{\mathbf{p}}\left(\hat{\mathbf{p}} \cdot \mathbf{S}_{H e}\right) B_{2, S_{H e}}^{\tau}(|\mathbf{p}|, E)$

Adding FSI, more terms should be included.

We are now evaluating the SSAs using the LF hadronic tensor, at finite values of $Q^{2}$. The preliminary results are quite encouraging, since, as shown in Table 1, LF longitudinal and transverse polarizations change little from the ones obtained within the NR spectral function and weakly differ from each other. Then we find that the extraction procedure works well within the LF approach as it does in the non relativistic case.

Concerning the FSI, we plan to include in our LF approach the FSI between the jet produced from the hadronizing quark and the two-nucleon system through the Glauber approach of Ref. [22].

\section{The $J=1 / 2$ LF spectral function and the nucleon LF TMDs}

The TMDs for a nucleon with total momentum $P$ and spin $S$ are introduced through the q-q correlator

$$
\begin{aligned}
\Phi(k, P, S)_{\alpha \beta}= & \int d^{4} z e^{i k \cdot z}\left\langle P S\left|\bar{\psi}_{q \beta}(0) \psi_{q \alpha}(z)\right| P S\right\rangle \\
= & \frac{1}{2}\left\{A_{1} \mathbb{P}+A_{2} S_{L} \gamma_{5} P+A_{3} P \gamma_{5} \$_{\perp}\right. \\
+ & \frac{1}{M} \widetilde{A}_{1} \vec{k}_{\perp} \cdot \vec{S}_{\perp} \gamma_{5} P+\widetilde{A}_{2} \frac{S_{L}}{M} P \gamma_{5} k_{\perp} \\
& \left.+\frac{1}{M^{2}} \widetilde{A}_{3} \vec{k}_{\perp} \cdot \vec{S}_{\perp} P \gamma_{5} k_{\perp}\right\}_{\alpha \beta},
\end{aligned}
$$

where $k$ is the parton momentum in the laboratory frame, so that the six twist-2 T-even functions, $A_{i}, \widetilde{A_{i}}(i=1,3)$, can be obtained by proper traces of $\Phi(k, P, S)$.

Indeed, particular combinations of the functions $A_{i}, \widetilde{A_{i}}(i=1,3)$ can be obtained by the following traces of $\Phi(k, P, S)$ :

$$
\begin{aligned}
& \frac{1}{2 P^{+}} \operatorname{Tr}\left(\gamma^{+} \Phi\right)=A_{1}, \\
& \frac{1}{2 P^{+}} \operatorname{Tr}\left(\gamma^{+} \gamma_{5} \Phi\right)=S_{L} A_{2}+\frac{1}{M} \vec{k}_{\perp} \cdot \vec{S}_{\perp} \widetilde{A_{1}}, \\
& \frac{1}{2 P^{+}} \operatorname{Tr}\left(\mathrm{i} \sigma^{\mathrm{j}+} \gamma_{5} \Phi\right)=S_{\perp}^{j} A_{3}+ \\
&+\frac{S_{L}}{M} k_{\perp}^{j} \widetilde{A_{2}}+\frac{1}{M^{2}} \vec{k}_{\perp} \cdot \vec{S}_{\perp} k_{\perp}^{j} \widetilde{A_{3}}(j=x, y) .
\end{aligned}
$$

The six T-even twist-2 TMDs for the quarks inside a nucleon can be obtained by integration of the functions
$A_{i}, \widetilde{A_{i}}$ on $k^{+}$and $k^{-}$as follows

$$
\begin{gathered}
f\left(x,\left|\mathbf{k}_{\perp}\right|^{2}\right)=\frac{1}{2} \int \frac{d k^{+} d k^{-}}{(2 \pi)^{4}} \delta\left[k^{+}-x P^{+}\right] 2 P^{+} A_{1}, \\
\Delta f\left(x,\left|\mathbf{k}_{\perp}\right|^{2}\right)=\frac{1}{2} \int \frac{d k^{+} d k^{-}}{(2 \pi)^{4}} \delta\left[k^{+}-x P^{+}\right] 2 P^{+} A_{2}, \\
g_{1 T}\left(x,\left|\mathbf{k}_{\perp}\right|^{2}\right)=\frac{1}{2} \int \frac{d k^{+} d k^{-}}{(2 \pi)^{4}} \delta\left[k^{+}-x P^{+}\right] 2 P^{+} \widetilde{A_{1}}, \\
\Delta_{T}^{\prime} f\left(x,\left|\mathbf{k}_{\perp}\right|^{2}\right)=\frac{1}{2} \int \frac{d k^{+} d k^{-}}{(2 \pi)^{4}} \delta\left[k^{+}-x P^{+}\right] 2 P^{+} \times \\
\left.h_{1 L}^{\perp}\left(x,\left|\mathbf{k}_{\perp}\right|^{2}\right)=\frac{1}{2} \int \frac{\left|\mathbf{k}_{\perp}\right|^{2}}{2 M^{2}} \widetilde{A_{3}}\right), \\
h_{1 T}^{\perp}\left(x,\left|\mathbf{k}_{\perp}\right|^{2}\right)=\frac{1}{2} \int \frac{d k^{+} d k^{-}}{(2 \pi)^{4}} \delta\left[k^{+}-x P^{+}\right] 2 P^{+} \widetilde{A_{2}},
\end{gathered}
$$

Let us consider the contribution to the correlation function from on-mass-shell fermions

$$
\begin{aligned}
& \Phi_{p}(k, P, S)=\frac{\left(k_{o n}+m\right)}{2 m} \Phi(k, P, S) \frac{\left(k_{o n}+m\right)}{2 m}= \\
= & \sum_{\sigma \sigma^{\prime}} u_{L F}\left(\tilde{k}, \sigma^{\prime}\right) \bar{u}_{L F}\left(\tilde{k}, \sigma^{\prime}\right) \Phi(k, P, S) u_{L F}(\tilde{k}, \sigma) \bar{u}_{L F}(\tilde{k}, \sigma)
\end{aligned}
$$

and let us identify $\bar{u}_{L F}\left(\tilde{k}, \sigma^{\prime}\right) \Phi(k, P, S) u_{L F}(\tilde{k}, \sigma)$, up to a kinematical factor $K$, with the LF nucleon spectral function, $\mathcal{P}_{\sigma^{\prime} \sigma}\left(\tilde{\kappa}, \epsilon_{S}, S\right)[14]$ :

$$
\bar{u}_{L F}\left(\tilde{k}, \sigma^{\prime}\right) \Phi(k, P, S) u_{L F}(\tilde{k}, \sigma)=K \mathcal{P}_{\sigma^{\prime} \sigma}\left(\tilde{\kappa}, \epsilon_{S}, S\right)
$$

In a reference frame where $\mathbf{P}_{\perp}=0$, the following relation holds between the off-mass-shell minus component $k^{-}$of the momentum of the struck quark and the spectator diquark energy $\epsilon_{S}$ :

$$
k^{-}=\frac{M^{2}}{P^{+}}-\frac{\left(\epsilon_{S}+m\right) 4 m+\left|\boldsymbol{k}_{\perp}\right|^{2}}{P^{+}-k^{+}}
$$

Let us approximate the full correlation function $\Phi(k, P, S)$ by its particle contribution $\Phi_{p}(k, P, S)$. Then, through relations analogous to the ones of Eq. (14), which allow one to obtain the functions $A_{i}, \widetilde{A_{i}}$ from the traces of $\Phi(k, P, S)$, the valence approximations $A_{i}^{V}, \widetilde{A}_{i}^{V}(i=1,3)$ for the functions $A_{i}, \widetilde{A_{i}}$ can be obtained by the traces $\quad\left[\gamma^{+} \Phi_{p}(k, P, S)\right], \quad\left[\begin{array}{lll}\gamma^{+} & \gamma_{5} & \Phi_{p}(k, P, S)\end{array}\right]$, and $\left[k_{\perp} \gamma^{+} \gamma_{5} \Phi_{p}(k, P, S)\right]$. However these same traces can be also expressed through the LF spectral function, since from Eqs. $(16,17)$ one has

$$
\operatorname{Tr}\left[\gamma^{+} \Phi_{p}(k, P, S)\right]=\frac{k^{+}}{m} K \operatorname{Tr}\left[\mathcal{P}\left(\tilde{\kappa}, \epsilon_{S}, S\right)\right]
$$




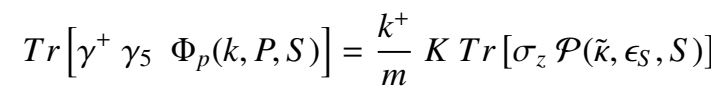

$\operatorname{Tr}\left[k_{\perp} \gamma^{+} \gamma_{5} \Phi_{p}(k, P, S)\right]=\frac{k^{+}}{m} \operatorname{Tr}\left[\boldsymbol{k}_{\perp} \cdot \boldsymbol{\sigma} \mathcal{P}\left(\tilde{\kappa}, \epsilon_{S}, S\right)\right]$

In turn, as in the ${ }^{3} \mathrm{He}$ case, the traces $\operatorname{Tr}(\mathcal{P} I), \operatorname{Tr}\left(\mathcal{P}_{\sigma_{z}}\right)$, $\operatorname{Tr}\left(\mathcal{P}_{\sigma_{i}}\right)(i=x, y)$ can be expressed in terms of three scalar functions, $B_{0}, B_{1}, B_{2}$ and known kinematical factors.

Then, within the LF approach with a fixed number of particles and in the valence approximations, the six leading twist T-even functions $A_{i}^{V}, \widetilde{A}_{i}^{V}(i=1,3)$ can be expressed in terms of the three independent scalar functions $B_{0}, B_{1}, B_{2}$. Therefore, in the valence approximation only three of the six T-even TMDs are independent [14].

\section{Conclusion}

A realistic study of the DIS processes off ${ }^{3} \vec{H} e$ and in particular of the SSAs in the reaction ${ }^{3} \vec{H} e\left(e, e^{\prime} \pi^{ \pm}\right) X$ beyond the PWIA and the non relativistic approach is under way.

The FSI effects are evaluated through the GEA and the introduction of a distorted spin-dependent spectral function. The relativistic effects are studied through the analysis of a LF spectral function (up to now only in PWIA).

Our preliminary results are quite encouraging. Indeed nuclear effects in the extraction of the neutron information were found to be under control, even when the interaction in the final state is considered, and the relativistic effects appear to be weak. An analysis at finite $Q^{2}$ with the LF spectral function is in progress in order to test the extraction procedure of the neutron information from ${ }^{3} \vec{H} e\left(e, e^{\prime} \pi^{ \pm}\right) X$ experiments, when both the FSI and the relativistic effects are considered. Our next step is to complete this program. Then we will apply the LF spectral function to other processes (e.g., DVCS) to exploit other possibilities to use ${ }^{3} \mathrm{He}$ as an effective neutron target and as a laboratory for light-front studies.

The introduction of a LF spin-dependent spectral function for a nucleon allowed us to find exact relations among the six leading twist T-even TMDs from general properties within LF dynamics with a fixed number of degrees of freedom and in the valence approximation. Then it can be shown that in the valence approximation only three of the six T-even TMDs are independent. The above relations are precisely predicted within LF dynamics, and could be experimentally checked to test the LF description of SiDIS in the valence region.

\section{References}

[1] V. Barone, A. Drago, P. G. Ratcliffe, Physics Reports 359, 1 (2002)

[2] D.W. Sivers, Physical Review D 41, 83 (1990); Physical Review D 43, 261 (1991)

[3] J.C. Collins, Nuclear Physics B 396, 161 (1993)

[4] S. Scopetta, Physical Review D 75, 054005 (2007)
[5] A. Airapetian et al., The HERMES Collaboration, Physical Review Letters 94, 012002 (2005)

[6] V. Yu. Alexakhin et al., COMPASS Collaboration, Physical Review Letters 94, 202002 (2005)

[7] A. Signori, A. Bacchetta, M. Radici, Int. J. Mod. Phys. Conf. Ser. 225, 9, (2024)

[8] G. Cates et al., E12-09-018, JLAB approved experiment, hallaweb.jlab.org/collab/PAC/PAC38// E1209-018-SIDIS.pdf

[9] A. Del Dotto, L. Kaptari, E. Pace, G. Salmè, S. Scopetta, Few-Body Syst. 55, 877 (2014) DOI 10.1007/s00601-014-0828-0

[10] A. Del Dotto, L. Kaptari, E. Pace, G. Salmè, S. Scopetta and E. Cisbani, EPJ Web of Conferences 73, 02019 (2014)

[11] A. Del Dotto, E. Pace, G. Salmè, S. Scopetta, Il Nuovo Cimento C 35, 101 (2012)

[12] E. Pace, A. Del Dotto, M. Rinaldi, G. Salmè, S. Scopetta, Few-Body Syst. 54, 1079 (2013)

[13] E. Pace, G. Salmè, S. Scopetta, A. Del Dotto, Acta Physica Polonica, B 6, 103 (2013)

[14] A. Del Dotto, E. Pace, G. Salmè, S. Scopetta, to be published

[15] C. Ciofi degli Atti, E. Pace, G. Salmè, S. Scopetta, Physical Review C 48, R968 (1993)

[16] C. Ciofi degli Atti et al., Physical Review C 46, R 159 (1992); A. Kievsky et al., Physical Review C 56, 64 (1997)

[17] K. Abe et al., E154 Collaboration, Physical Review Letters 79, 26 (1997)

[18] R. B. Wiringa, V. G. J. Stoks and R. Schiavilla, Physical Review C 51, 38 (1995)

[19] X. Qian et al., Jefferson Lab Hall A Collaboration, Physical Review Letters 107, 072003 (2011)

[20] K. Allada et al., Jefferson Lab Hall A Collaboration, Physical Review C 89, 042201 (2014)

[21] L. Kaptari, A. Del Dotto, E. Pace, G. Salmè, S. Scopetta, Physical Review C 89, 035206 (2014)

[22] C. Ciofi degli Atti, L. Kaptari, Physical Review C 83, 044602 (2011)

[23] S. Kuhn, C. Keppel, W. Melnitchouk (spokespersons), et al., "The structure of the free neutron via spectator tagging", www.jlab.org/exp_prog/proposals/03prop.html (PR03-012.pdf); www.jlab.org/exp_prog/proposals/06/PR12-06113.pdf

[24] C. Ciofi degli Atti, L. P. Kaptari and B. Z. Kopeliovich, Eur. Phys. J. A 19, 145 (2004)

[25] X. Jiang et al., www.jlab.org/exp_prog/generated/ apphalla.html; J. Arrington et al., www.jlab.org/ jinhuang/12GeV/12GeVLongitudinalHe3.pdf.

[26] P.A.M. Dirac, Rev. Mod. Phys. 21, 392 (1949)

[27] B. Bakamjian and L.H. Thomas, Physical Review 92 , $1300(1953)$ 